\section{REGISTER AS A LIVING ORGAN DONOR}

As many as 1,000 people die waiting for an organ transplant in the UK each year. 8-14 July 2013 was National Transplant Week in the UK. In recognition of this event MatchingDonors, MatchingDonors.co.uk, MatchingDonors.com and MatchingDonors.org, a 501c3 nonprofit organisation and the world's largest online living donor organisation, launched a public education campaign throughout the UK to help people recognise that they can be a living organ donor and to encourage them to register as an altruistic living organ donor.

The MatchingDonors Living Organ Donor Initiative programme has already saved thousands of lives. All MatchingDonors, MatchingDonors. com, MatchingDonors.org, and MatchingDonors.co.uk services and memberships are totally free to all patients and donors in the UK.

To find out more, visit www. matchingdonors.co.uk.

\section{CPD SESSIONS TO SUIT YOU AND YOUR PRACTICE}

dbg offers a superb range of training courses designed to cater for all your training and CPD needs. These include:

- Medical emergencies

- Infection control

- Basic fire safety awareness

- Manual handling

- CoshH \& legionella awareness

- Dental radiography \&t radiation protection

- Bespoke handpiece user care

- User validation training in line with HTM 01-05.

Each of dbg's courses has been designed to be highly relevant. Call dbg on 01606861950 or visit www.thedbg.co.uk.

\section{TWO MINUTES TO SAVE A LIFE}

The Mouth Cancer Foundation has devised a 'Two minutes to save a life' screening protocol, a 10-point checklist that every dentist should be able to include in their routine examinations.

The Mouth Cancer Foundation accredits practices that actively prove they regularly carry out thorough head and neck cancer screenings and operate a documented referral pathway with a local specialist department. Full membership includes access to a dedicated section of the charity website and free one hour CPD element as well as professional development and training modules suitable for all members of the practice team. www.mouthcancerfoundation. org/mcsas/about-mcsas

\section{TAKING NEEDLESTICK INJURIES SERIOUSLY}

Plandent take needlestick injuries very seriously, as it's been reported that up to 56\% of dental surgeons suffer at least one incidence every year. Plandent is pleased to announce that they are now supplying inSafe contaminated needle disposal system from Aztec to help combat this issue.

inSafe provides complete protection from the beginning of the medical procedure, through to the disposal of the needle. The specially designed syringe protects the needle at all times and its partnering sharps container removes the contaminated needle in complete safety.

The needle is protected by a lockable sleeve ensuring safety for the operator, and with a one-handed disposal of the contaminated needle this further reduces any risk. The autoclaveable inSafe syringe looks and feels like a conventional syringe, allowing a choice of aspiration techniques, and you can change the cartridge during the procedure without compromise. The inSafe system is economical with minimal disposable components.

For further information or to request your free full-colour comprehensive catalogue contact Plandent on Freecall 0500 500322.

\title{
STAY SAFE AND COMPLIANT WITH SUSAN
}

The Control of Substances Hazardous to Health Regulations (COSHH) 2002 state that you must be committed to the provision and maintenance of a safe and healthy working environment for all staff and visitors to the practice. You must also seek to eradicate or minimise any risk of harm caused by hazardous substances.

There is now an app that will help keep your dental practice up to date and compliant. The

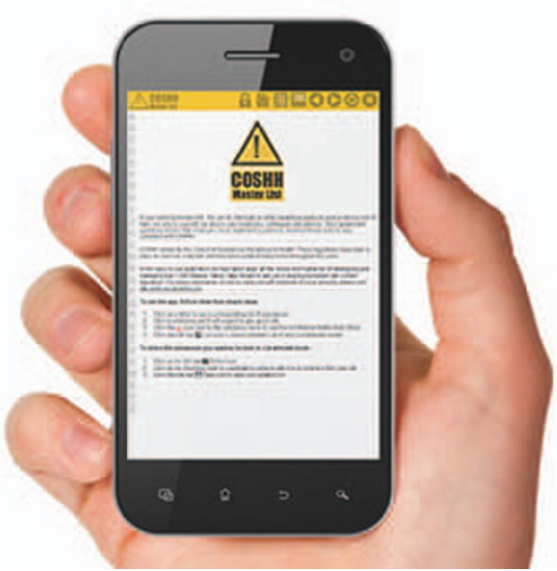

SUSAN COSHH APP will save you hours of effort whilst keeping you up to date and compliant with the current laws as follows:

- SUSAN has thousands of Material Safety Data Sheets and commonly used substances from manufacturers in the system. All you will have to do is highlight the ones you use at your practice

- Helps meet and gather evidence for essential CQC Outcomes

- An interactive risk assessment tool for each COSHH substance

- SUSAN stores data sheets in the cloud so you and your team can access them from any internetconnected device, whenever you need

- Easy to read format, means your team will quickly become familiar with everything they need to know about COSHH

- SUSAN's team of experts continually update content so that you don't have to.

COSHH is just one small part of the vast amount of Health \& Safety issues that are part of managing a dental practice. SUSAN has all of these covered in a constantly updated easy to read format to save you time and stress. www.susandental.com 